\title{
¿INFLUYE EL AULA INVERTIDA EN LA MOTIVACIÓN Y EL RENDIMIENTO ACADÉMICO DE ESTUDIANTES UNIVERSITARIOS?
}

How does flipped classroom influence the motivation and academic performance of university students?

Como a flippedclassroom influence a motivação e desempenho acadêmico dos estudantes universitários?

Plan de Apoyo a los Grupos de Innovación Docente de la ULE (PAGID 2016). Título del Proyecto financiado: "Propuesta de puesta en marcha de la metodología activa Flipped Classroom para fomentar la participación y motivación de los estudiantes de Grado"

\section{Cristina Mendaña Cuervo (1)}

\section{Raquel Poy Castro (2)}

\section{Anselma González Fernández (3)}

\section{$M^{a}$ Victoria Arana Suárez (4)}

\section{Enrique López González (5)}

(1) Universidad de León, España. Teléfono: +34987291742. Correo: cristina.mendana@unileon.es

(2) Universidad de León, España. Teléfono: +34987295137. Correo: rpoyc@unileon.es.

(3) Universidad de León, España. Teléfono: +34987293025. Correo: anselma.gonzalez@unileon.es

(4) Universidad de León, España. Teléfono: +34987291467. Correo: mvaras@unileon.es

(5) Universidad de León, España. Teléfono: +34987291742. Correo: enrique.lopez@unileon.es

\begin{abstract}
Resumen
El objetivo de este estudio de investigación ha sido analizar cómo la aplicación del aula invertida puede influir en la motivación y/o en los resultados académicos de alumnos pertenecientes a una misma rama de conocimiento, en tres Grados distintos de la ULE. ¿Influye el aula invertida en la motivación y el rendimiento académico de estudiantes universitarios?
\end{abstract}


Los alumnos de las asignaturas fueron distribuidos al azar en un grupo experimental GE- (sometido a la nueva metodología) y un grupo control -GC- al que se le aplicó la metodología tradicional. Para el contraste de hipótesis y su análisis se recurrió a técnicas estadísticas. Los resultados evidencian una diferencia significativa entre el GE y el GC en relación a la motivación en el caso de asignaturas de carácter obligatorio o de formación básica, pero no en optativas. En cuanto al rendimiento académico no se ha observado diferencias significativas en ningún caso.

Palabras clave: Motivación; rendimiento académico; educación superior

\begin{abstract}
The aim of this study was to analyze how the Flipped Classroom methodology can influence the student's motivation and academic performance, belonging to the same knowledge field, in three different degrees the ULE. The students of subjects were randomly distributed in an experimental group -EG- (to which the new methodology was applied) and a control group-CG- (to which the traditional methodology was applied). To contrast the research hypotheses and their analyses, statistical measurements were used. The results show a significant difference between EG and CG in relation to motivation, in the case of compulsory subjects or basic training, but not in optional subject. However as to academic performance, no significant differences were observed in any case.
\end{abstract}

Keywords: Motivation, academic performance, higher education

\title{
Resumo
}

O objetivo desta experiência educacional foi implementar uma metodologia ativa (Flipped Classroom), a fim de verificar o seu impacto na motivação e desempenho acadêmico dos estudantes. Pupilas do "Teoria e instituições contemporâneas de Educação" sujeito Mestrado na Infância foram distribuídos aleatoriamente em um grupo experimental -GE- (submetido a nova metodologia) e um grupo de controlo ao qual aplicado o -GC- metodologia tradicional. Para os testes de hipóteses e análise, foi usado técnicas estatísticas paramétricas. Os resultados mostram uma diferença significativa entre GE e GC emr elação à motivação, mas não em relação ao desempenho acadêmico. 
Estes resultados podem ser explicados com base nas informações obtidas durante o curso desta experiência.

Palavras-chave: Motivação; rendimento acadêmico; Educação superior

\section{Introducción}

El objetivo del estudio de investigación es someter a contraste si la metodología Flipped Classroom-FC- (Bergmann \& Sams, 2012) aplicada a diferentes Grados de una misma rama de conocimiento, en diferentes cursos y en asignaturas con distinto carácter (obligatoria/optativa/formación básica) arroja resultados similares en relación a la motivación y/o rendimiento académico de los estudiantes.

\section{Método}

Se analizan los datos del diseño experimental de las asignaturas participantes en el estudio de investigación (Tabla 1), asignando al azar los estudiantes en dos grupos (grupo experimental GE y grupo de control GC y). En el primer caso se aplica la metodología objeto de estudio, mientras que en el GC se utiliza la metodología tradicional.

Tabla 1.

Muestras de conveniencia

\begin{tabular}{|c|c|c|c|c|c|}
\hline & Asignatura & Estudios & Carácter & Curso & ECTs \\
\hline A1 & Contabilidad de Costes & GADE & Obligatoria & $3^{\circ}$ & 6 \\
\hline A2 & $\begin{array}{l}\text { Marco Jurídico Profesional de la } \\
\text { Actividad Física y el Deporte }\end{array}$ & GCAFyD & Optativa & $3^{\circ}-4^{0}$ & 6 \\
\hline A3 & $\begin{array}{l}\text { Teoría e Instituciones } \\
\text { Contemporáneas de la Educación }\end{array}$ & GEI & FB & $1^{\mathrm{o}}$ & 6 \\
\hline
\end{tabular}

Al finalizar la experiencia ambos grupos fueron sometidos a una misma prueba de evaluación (Tabla 2) para recabar los datos de las variables objeto de análisis.

Tabla 2.

Alumnos presentados a las pruebas de evaluación

\begin{tabular}{lcccccc}
\hline \multirow{2}{*}{ Asignatura } & \multicolumn{3}{c}{ Alumnos } & \multicolumn{2}{c}{ Presentados } & \multicolumn{2}{c}{$\%$} \\
\cline { 2 - 7 } & GE & GC & GE & GC & GE & GC \\
\hline A1 & 49 & 73 & 41 & 48 & 0,83674 & 0,65753 \\
A2 & 18 & 23 & 16 & 18 & 0,8888 & 0,78608 \\
A3 & 43 & 49 & 43 & 44 & 1,00 & 0,89796 \\
\hline
\end{tabular}

Las hipótesis de investigación sometidas a contraste se plantean en los siguientes términos: 
Hipótesis de investigación 1. La experiencia de aplicar FC mejora la motivación de los alumnos

$\mathrm{H}_{0}$ : La proporción de presentados es la misma en el GC y en el GE

$\mathrm{H}_{1}$ : La proporción de presentados no es la misma en el GC y en el GE

Hipótesis de investigación 2. El rendimiento académico de los estudiantes se incrementa tras la experiencia de aplicar FC

$\mathrm{H}_{0}$ : Las calificaciones son iguales el GC y en el GE

$\mathrm{H}_{1}$ : Las calificaciones no son iguales el GC y en el GE

\section{Resultados y discusión}

Hipótesis de investigación 1. El resultado del contraste de hipótesis de diferencia de proporciones para cada materia se muestra en la Tabla 3.

Tabla 3.

Resultados contraste hipótesis

\begin{tabular}{lcc}
\hline & Estadístico Z & p-valor \\
\hline A1 & 2,1843 & 0,01446 \\
A2 & 0,89754 & 0,1847 \\
A3 & 2,15405 & 0,01561 \\
\hline
\end{tabular}

Los resultados obtenidos, para un nivel de significación de 5\%, permiten afirmar que:

A1. Se rechaza $\mathrm{H}_{0}$, asumiendo por tanto que la proporción de alumnos presentados en el GE es significativamente mayor que en el GC.

A2. En este caso, no hay diferencias significativas en el número de alumnos presentados entre los dos grupos.

A3. También se rechaza $\mathrm{H}_{0}$, asumiendo que la proporción de alumnos presentados del GE (el 100\%) es significativamente mayor que en el GC.

En consecuencia, la motivación de los alumnos en el caso de asignaturas obligatorias o de formación básica ha mejorado, mientras que en la asignatura optativa no se observa esta mejoría

Hipótesis de investigación 2. En primer lugar, se han sometido las calificaciones de los dos grupos en cada asignatura a las pruebas de normalidad (Tabla 4), con el fin de conocer si es factible la aplicación de una técnica paramétrica para el contraste. 
Por tanto, para la comparación de medias es posible realizar la prueba paramétrica tStudent en las materias A1 y A3, mientras que para la materia A2 al no poder asumir normalidad, se ha optado por aplicar la técnica no paramétrica U de Mann-Whitney para la comparación de medianas. Los resultados se muestran en las Tablas 5 y 6.

Tabla 4.

Pruebas de normalidad

\begin{tabular}{|c|c|c|c|c|c|c|c|}
\hline & & \multicolumn{3}{|c|}{ Kolmogorov-Smirnov ${ }^{\mathrm{a}}$} & \multicolumn{3}{|c|}{ Shapiro-Wilk } \\
\hline & & Estadístico & gl & Sig. & Estadístico & gl & Sig. \\
\hline "GC" & \multirow{2}{*}{ A1 } & ,080 & 48 & ,200 & ,969 & 48 & ,222 \\
\hline "GE" & & ,072 & 41 & ,200 & ,988 & 41 & ,943 \\
\hline "GC" & \multirow{2}{*}{ A2 } & ,207 & 17 & ,052 & ,901 & 17 & ,071 \\
\hline "GE" & & ,288 & 17 & ,001 & ,870 & 17 & ,022 \\
\hline "GC" & \multirow{2}{*}{ A3 } & ,096 & 44 & ,200 & 963 & 44 & ,164 \\
\hline "GE" & & ,084 & 43 & ,200 & ,977 & 43 &, 521 \\
\hline
\end{tabular}

a. Corrección de significación de Lilliefors

Tabla 5.

Comparación de medias (A1 y A3)

\begin{tabular}{|c|c|c|c|c|c|c|c|c|c|c|}
\hline & & \multicolumn{2}{|c|}{$\begin{array}{l}\text { Prueba de } \\
\text { Levene }\end{array}$} & \multicolumn{7}{|c|}{ prueba t para la igualdad de medias } \\
\hline & & & & & & Sig. & $\begin{array}{l}\text { Diferen } \\
\text { cia de }\end{array}$ & $\begin{array}{c}\text { Diferenci } \\
\text { a de error }\end{array}$ & $\begin{array}{r}95 \% \text { de in } \\
\text { confian } \\
\text { difer }\end{array}$ & $\begin{array}{l}\text { ervalo de } \\
\text { a de la } \\
\text { ncia }\end{array}$ \\
\hline & & $\mathrm{F}$ & Sig. & $\mathrm{t}$ & $\mathrm{gl}$ & (bilateral) & medias & estándar & Inferior & Superior \\
\hline \multirow{2}{*}{ A1 } & $\begin{array}{l}\text { Se asumen } \\
\text { varianzas iguales }\end{array}$ & ,003 & ,954 & $-1,217$ & 86 & ,227 &,- 76100 & ,62539 & $-2,00423$ & ,48224 \\
\hline & $\begin{array}{l}\text { No se asumen } \\
\text { varianzas iguales }\end{array}$ & & & $-1,220$ & 85,143 & ,226 &,- 76100 & ,62376 & $-2,00116$ & ,47917 \\
\hline \multirow{2}{*}{ A3 } & $\begin{array}{l}\text { Se asumen } \\
\text { varianzas iguales }\end{array}$ & 1,540 & ,218 & $-1,041$ & 85 & 301 &,- 31734 & ,30476 & $\begin{array}{c}- \\
, 92329\end{array}$ & ,28861 \\
\hline & $\begin{array}{l}\text { No se asumen } \\
\text { varianzas iguales }\end{array}$ & & & $-1,039$ & 81,806 & ,302 &,- 31734 & ,30538 & $\begin{array}{c}- \\
, 92486\end{array}$ & ,29019 \\
\hline
\end{tabular}

Tabla 6.

Comparación de medianas (A2)

\begin{tabular}{lr}
\hline U de Mann-Whitney & 131,000 \\
\hline W de Wilcoxon & 284,000 \\
\hline$Z$ &,- 484 \\
\hline Sig. asintótica (bilateral) &, 628 \\
\hline Significación exacta [2*(sig. unilateral)] &, $658^{\mathrm{b}}$ \\
\hline
\end{tabular}

Los resultados obtenidos, para un nivel de significación de 5\%, permiten afirmar que en todos los casos (A1, A2 y A3) se acepta $\mathrm{H}_{0}$, asumiendo, por tanto, que no se 
observan diferencias significativas en el rendimiento académico de los grupos experimental y control

\section{Conclusiones}

Los resultados del estudio de investigación permiten concluir, en relación a las hipótesis planteadas, que la motivación de los alumnos en el caso de asignaturas obligatorias o de formación básica ha mejorado, mientras que en la asignatura optativa no se observa esta mejoría. Estas conclusiones son plausibles teniendo en cuenta el diferente carácter de las materias, dado que asumimos que los alumnos de asignaturas optativas presentan una motivación intrínseca al realizar la elección de la misma. Sin embargo, en el resto de asignaturas la motivación es extrínseca y, por tanto, la metodología utilizada ha revertido un aumento en la misma.

En relación a los resultados académicos no se observa mejoría significativa en ningún caso. Ante la ausencia de mayor evidencia en la explotación del estudio desarrollado, las líneas de investigación a explorar se centrarían en realizar un análisis cualitativo de las respuestas de los alumnos a los cuestionarios creados ad hoc para esta experiencia (ya administrados a las muestras participantes en este estudio), y que excede los límites de este trabajo.

\section{Referencias}

Al-Zahrani, A. M. (2015). From passive to active: The impact of the flipped classroom through social learning platforms on higher education students' creative thinking. British Journal of Educational Technology, 46, 1133-1148. doi:10.1111/bjet.12353

Argente, E., García-Fornes, A., \& Espinosa, A. (2016). Aplicando la metodología Flipped-Teaching en el Grado de Ingeniería Informática: Una experiencia práctica. Actas de las XXII Jenui, Almería, 221-228. doi: http://hdl.handle.net/10251/78306

Bergmann, J., \& Sams, A. (2012). Flip your classroom: Reach every student in every class every day. Washington, DC: International Society for Technology in Education. 
Caviglia-Harris, J. (2016). Flipping the Undergraduate Economics Classroom: Using Online Videos to Enhance Teaching and Learning. Southern Economic Journal, 83,321-331. doi:10.1002/soej.12128.

Cheng, X., Ka Ho Lee, K., Chang, E. Y., \&Yang, X. (2016). The “flipped classroom” approach: Stimulating positive learning attitudes and improving mastery of histology among medical students. American Association of Anatomists. doi:10.1002/ase.1664

Sánchez Vera, M., Solano Fernández, I.M., \& González Calatayud, V. (2016). FLIPPEDTIC: Una experiencia de Flipped Classroom con alumnos de Magisterio. Revista Latinoamericana de Tecnología Educativa, 15(3), 69-81. doi: 10.17398/1695288X.15.3.69 